\title{
Triples of Sixth Powers With Equal Sums
}

\author{
By Simcha Brudno
}

\begin{abstract}
The diophantine equation $x^{6}+y^{6}+z^{6}=u^{6}+v^{6}+w^{6}$ is shown to have a two-parameter solution which is homogeneous of degree four. The solution also satisfies $x^{2}+y^{2}+z^{2}=u^{2}+v^{2}+w^{2}$; and in addition, $3 x+y+z=3 u+$ $v+w$.
\end{abstract}

The diophantine equation

$$
x^{6}+y^{6}+z^{6}=u^{6}+v^{6}+w^{6}
$$

is a particular instance of the much-studied problem of finding equal sums of like powers of integers, surveyed by Lander, Parkin and Selfridge in 1967 [4]. The smallest nontrivial solution was published by Subba Rao in 1934 [5], namely, $3^{6}+19^{6}+$ $22^{6}=10^{6}+15^{6}+23^{6}$. Early editions of Hardy and Wright [3] referred to this result as "an isolated curiosity". However, Lander, Parkin and Selfridge [4] discovered that (1) has ten primitive solutions in the range up to $2.5 \times 10^{14}$, and that all but one of these also satisfy

$$
x^{2}+y^{2}+z^{2}=u^{2}+v^{2}+w^{2} .
$$

In [1] it was shown that there are infinitely many primitive solutions to (1), each also satisfying (2) and

$$
v=y-z, \quad w=y+z
$$

Subsequently, in [2] the complete solution to (1), (2) and (3) was obtained in terms of an infinite cyclic group of rational points on a cubic curve. (Regrettably, the solution $5 P$ appeared in [2] with transcription errors in the values of $x$ and $w$; it should read $x=165809277507, y=151561337462, z=23038103009, u=$ $63175337782, v=128523234453$ and $w=174599440471$.)

The principal aim of this paper is to exhibit the following explicit solution to (1) in terms of parameters $m, n$ :

$$
\begin{aligned}
x & =2 m^{4}+4 m^{3} n-5 m^{2} n^{2}-12 m n^{3}-9 n^{4}, \\
y & =3 m^{4}+9 m^{3} n+18 m^{2} n^{2}+21 m n^{3}+9 n^{4}, \\
z & =-m^{4}-10 m^{3} n-17 m^{2} n^{2}-12 m n^{3}, \\
u & =m^{4}-3 m^{3} n-14 m^{2} n^{2}-15 m n^{3}-9 n^{4}, \\
v & =3 m^{4}+8 m^{3} n+9 m^{2} n^{2}, \\
w & =2 m^{4}+12 m^{3} n+19 m^{2} n^{2}+18 m n^{3}+9 n^{4} .
\end{aligned}
$$

This solution also satisfies (2); and in addition,

$$
3 x+y+z=3 u+v+w .
$$

Received July 28, 1975.

AMS (MOS) subject classifications (1970). Primary 10B15; Secondary 10B10, 10C10, $10 \mathrm{~J} 05$. 
TABLE 1

\begin{tabular}{rr|rr|rrr|rrr|r|r}
\hline$m$ & $n$ & $m^{\prime}$ & $n^{\prime}$ & $x$ & $y$ & $z$ & $u$ & $v$ & $w$ & $d$ & $d^{\prime}$ \\
\hline 0 & 1 & 1 & -1 & -1 & 1 & 0 & -1 & 0 & 1 & 9 & 4 \\
1 & 0 & 3 & -1 & 2 & 3 & -1 & 1 & 3 & 2 & 1 & 36 \\
1 & 1 & 3 & -2 & -1 & 3 & -2 & -2 & 1 & 3 & 20 & 45 \\
2 & -1 & 3 & 1 & -1 & 3 & 4 & 1 & 4 & -3 & 5 & 180 \\
1 & -2 & 3 & -5 & -74 & 33 & 47 & -73 & 23 & 54 & 1 & 36 \\
1 & 2 & 9 & -7 & -50 & 81 & -37 & -65 & 11 & 78 & 5 & 180 \\
2 & 1 & 9 & -5 & 11 & 243 & -188 & -103 & 148 & 249 & 1 & 36 \\
1 & -3 & 3 & -4 & -23 & 15 & 10 & -22 & 3 & 19 & 20 & 45 \\
1 & 3 & 6 & -5 & -271 & 372 & -127 & -317 & 27 & 356 & 4 & 9 \\
2 & -3 & 3 & -7 & -65 & 15 & 52 & -67 & 36 & 37 & 5 & 180 \\
4 & -1 & 9 & -1 & 43 & 81 & 32 & 55 & 80 & -3 & 5 & 180 \\
1 & -4 & 9 & -11 & -326 & 243 & 107 & -311 & 23 & 282 & 5 & 180 \\
3 & 2 & 5 & -3 & -26 & 225 & -169 & -121 & 111 & 230 & 9 & 4 \\
5 & -1 & 6 & -1 & 169 & 276 & 65 & 179 & 275 & 36 & 4 & 9 \\
5 & 1 & 9 & -4 & 389 & 891 & -590 & -46 & 775 & 831 & 4 & 9 \\
4 & -3 & 3 & 5 & -409 & 93 & 512 & -293 & 528 & -271 & 1 & 36 \\
5 & -2 & 9 & 1 & 86 & 729 & 655 & 431 & 775 & -426 & 1 & 36 \\
6 & 1 & 7 & -3 & 71 & 147 & -92 & 1 & 132 & 133 & 45 & 20 \\
7 & -1 & 9 & -2 & 163 & 243 & 14 & 142 & 245 & 75 & 20 & 45 \\
\hline & & & & & & & & & & & \\
\hline
\end{tabular}

Of the ten smallest primitive solutions to (1), listed in [4], all but the sixth satisfy (2). Only the second satisfies (3), while (4) gives rise to all except the seventh (and, of course, the sixth).

It should be noted that a particular choice of $m$ and $n$ does not necessarily yield a primitive solution in (4), even if $m$ and $n$ are coprime. Indeed, suppose $(m, n)=1$ and $d=(x, y, z, u, v, w)$. It is not difficult to prove that (i) $2 \mid d$ just if $m \equiv n$ $(\bmod 2)$, and then $2^{2} \| d$; (ii) $3 \mid d$ just if $m \equiv 0(\bmod 3)$, and then $3^{2} \| d$; and (iii) $5 \mid d$ just if $m \equiv n$ or $2 m \equiv n(\bmod 5)$, and then $5^{1} \| d$. Moreover, suppose $p \mid d$ for some prime $p>5$. Clearly, $p \nmid m n$, so $v \equiv 0(\bmod p)$ yields $3 m^{2}+8 m n+9 n^{2} \equiv 0(\bmod p)$. With $y-v \equiv 0(\bmod p)$ this leads to $10(m+3 n) \equiv 0(\bmod p)$, and finally with $z \equiv$ $0(\bmod p)$ this yelds $72 n^{4} \equiv 0(\bmod p)$, which is impossible. Hence, $d$ has no prime factor greater than 5 .

Consider the transformation to (4) which results from replacing $m, n$ by $m^{\prime}, n^{\prime}$ satisfying

$$
m^{\prime}: n^{\prime}=-3(m+n):(m+3 n) .
$$

If $x, y, z, u, v, w$ is the solution corresponding to $m, n$ and $x^{\prime}, y^{\prime}, z^{\prime}, u^{\prime}, v^{\prime}, w^{\prime}$ is the solution corresponding to $m^{\prime}, n^{\prime}$, then

$$
x^{\prime}: y^{\prime}: z^{\prime}: u^{\prime}: v^{\prime}: w^{\prime}=u: v: w: x: y: z .
$$


It follows that any particular primitive solution to (1), (2) and (5) obtained from (4) actually arises from two distinct ratios $m: n$.

Next, we remark that any solution to (1), (2) and (5) has an interesting geometrical interpretation. The points $(x, y, z)$ and $(u, v, w)$ in $E^{3}$ are lattice points which simultaneously lie on a sphere $X^{2}+Y^{2}+Z^{2}=a$, a concentric closed surface $X^{6}+$ $Y^{6}+Z^{6}=b$, and a double cone with vertex at the origin and axis in the direction $3: 1: 1$. It is intriguing to speculate that the solutions might turn out to have some physical interpretation.

Finally, in Table 1 are listed all primitive solutions obtained from (4) with the property that $\max \{|x|,|y|,|z|\}<10^{3}$. As remarked earlier, these include all but two of the numerical examples given in Table IX of [4].

Acknowledgments. The author wishes to thank R. B. Eggleton and J. L. Selfridge for assistance in writing up these results, and Denis Bartley without whose help the computations could not have been performed.

901 Ashland Avenue

Chicago, Illinois 60607

1. SIMCHA BRUDNO, "On generating infinitely many solutions of the Diophantine equation $A^{6}+B^{6}+C^{6}=D^{6}+E^{6}+F^{6}$," Math. Comp, v. 24, 1970, pp. 453-454. MR 42 \#5903.

2. SIMCHA BRUDNO \& IRVING KAPLANSKY, "Equal sums of sixth powers," J. Number Theory, v. 6, 1974, pp. 401-403.

3. G. H. HARDY \& E. M. WRIGHT, An Introduction to the Theory of Numbers, 3rd ed., Clarendon Press, Oxford, 1954, Chapter 21 (the comment is dropped in the 4th ed.). MR 16, 673.

4. L. J. LANDER, T. R. PARKIN \& J. L. SELFRIDGE, "A survey of equal sums of like powers," Math. Comp., v. 21, 1967, pp. 446-459. MR 36 \#5060.

5. K. SUBBA RAO, "On sums of sixth powers," J. London Math. Soc., v. 9, 1934, pp. 172-173. 